\title{
Vegetais cultivados em quintais rurais Piauienses com indicação anticâncer: uma busca pelo conhecimento tradicional
}

\author{
Maria Carolina de Abreu, Paulo Henrique da Silva e Ykaro Richard Oliveira \\ Universidade do Piauí, PI, Brasil \\ mariacarolinabreu@hotmail.com; paulohenriquemh1@gmail.com; ykr-oliveira@hotmail.com
}

\section{Resumo}

Objetivou-se neste estudo, identificar as plantas medicinais com indicação anticâncer cultivadas em quintais pelos moradores da comunidade rural de Aroeiras, Monsenhor Hipólito-PI, Brasil. Foi realizada uma pesquisa de campo de abordagem quali-quantitativa no período de fevereiro a maio de 2014, na qual foram executadas entrevistas semiestruturadas aos moradores selecionados através da amostragem não-probabilística "bola de neve", sendo utilizada a técnica da turnê-guiada no acompanhamento dos quintais e, simultâneo ao levantamento de dados, foram coletadas amostras das plantas citadas, as quais foram herborizadas segundo técnicas usuais em taxonomia vegetal. Ao todo, foram averiguados 71 quintais, com uma amostra de 71 entrevistados, onde 38,02\% reportaram cultivar algum vegetal indicado à prevenção ou tratamento do câncer. Logo, nos quintais pesquisados foram levantadas seis espécies: Morinda citrifolia L. (55,55\%), Aloe vera (L.) Burm. F. (37,03\%), Annona muricata L. (14,81\%), Euphorbia tirucalli L. (11,11\%), Chenopodium ambrosioides L. (3,70\%) e Capsicum frutescens L. $(3,70 \%)$, constatando-se desta forma, a persistência da cultura do cultivo de vegetais com finalidades medicinais na localidade estudada. Entretanto, é notável a necessidade de esclarecimentos sobre o perigo do uso indiscriminado de tais vegetais como prevenção ao agravamento das enfermidades.

Palavras-chave: Etnobotânica; Plantas medicinais; Tratamento do câncer; Neoplasia.

\section{Abstract}

The aim of this study was to identify the medicinal plants with anticancer indication grown in backyards by residents of the rural community Aroeiras, Monsenhor Hipolito - PI, Brazil. It was conducted a field research with quali-quantitative approach in the period February to May 2014, in which were executed semi-structured interviews with selected residents by sampling "snowball". The tour-guided technique was used in monitoring of backyards and simultaneous to the data collection, samples were collected from the mentioned plants, which were herborized according to standard techniques in plant taxonomy. In all, they investigated 71 backyards, with a sample of 71 respondents, where $38.02 \%$ reported cultivate some vegetable indicated for the prevention or treatment of cancer. Thus, in backyards were raised six species: Morinda citrifolia L. (55.55\%), Aloe vera (L.) Burm. F. (37.03\%), Annona muricata L (14.81\%), Euphorbia tirucalli L. (11.11\%), Chenopodium ambrosioides L. (3.70\%) and Capsicum frutescens L. (3.70\%), noting in this way, the persistence of vegetable cultivation for medicinal purposes in the locality studied. However, is remarkable the need for clarification of the danger of indiscriminate use of such vegetables as the prevention the worsening of the disease.

Key-words: Cancer treatment; Ethnobotany; Medicinal plants; Neoplasia. 


\section{Introdução}

As populações humanas interagem com uma grande variedade de espécies de plantas, desenvolvendo modos particulares de explorá-las para diversos fins, utilizando-as como alternativa de sobrevivência. Dentre estas, do repertório cultural, destaca-se o conhecimento acerca do uso de vegetais para finalidades terapêuticas (OLIVEIRA et al., 2010). O uso de produtos naturais pela humanidade ocorre desde tempos imemoriais; a busca por alívio e cura de enfermidades pela ingestão de ervas e folhas talvez tenha sido uma das primeiras maneiras de uso destes produtos (VIEGAS JR. et al. 2006), entretanto, de acordo com Lorenzi (2002), a utilização de plantas dentro do contexto medicinal tem evoluído no decurso do tempo, desde as formas mais simples de tratamento local até formas tecnologicamente sofisticadas de produção industrial utilizadas pelo homem moderno.

As pessoas tendem a utilizar uma ampla diversidade de plantas provenientes de diversos sítios ecológicos naturais ou manejados pelo ser humano, tais como áreas de vegetação nativa, zonas antrópicas e inteiramente descaracterizadas, e quintais (ALBUQUERQUE \& ANDRADE, 2002). Na zona rural, o uso de vegetais medicinais sempre existiu, onde os povos que nestas áreas habitam sustentam uma interação harmoniosa com o meio natural, pois dele retiram alimento, abrigo e, sobretudo, remédios para aliviar as dores ou se curar de alguma enfermidade. Estas pessoas que sempre residiram no campo apresentam vastos saberes acerca da maneira correta de usar as plantas medicinais além de estarem em contínua e recíproca troca de saberes (AMOROZO, 2002).

Nos dias de hoje, a terapêutica dos cânceres, em sua grande maioria, é considerada como uma das dificuldades mais desafiadoras do campo da medicina. De acordo com a localização, a extensão e o tipo de neoplasia, é possível optar por um ou por uma associação de tratamentos, dentre os quais se destacam a cirurgia, a quimioterapia e a radioterapia. Porém, observa-se o crescimento na procura de tratamentos "alternativos e/ou complementares" para o câncer, pelos pacientes, junto aos médicos. E, caso não seja acatado em sua solicitação, o paciente procura por conta própria ou com ajuda de familiares, amigos e/ou vizinhos, outras maneiras de tratamento, focando a utilização de espécies vegetais (ARAUJO et al., 2007; BRASIL, 2009).

Fonseca e Fioravanti (1995) abordam que as alternativas mais rápidas e baratas são as plantas, uma vez que estas sintetizam substâncias químicas que podem ser utilizadas como remédios. Medicações usadas com sucesso no tratamento alopático do câncer hoje são oriundas da natureza, como por exemplo, os alcaloides da vinca (a vincristina, a vindesina e a vimblastina), cujos são provenientes da Lochnera rosea (L.) Rchb. ex K. Schum., conhecida popularmente como vinca rosa. Plantas como a Taxus brevifolia Nutt., são consideradas como singulares, visto que nenhum químico é capaz de copiar; é dela que se extrai o taxol, eficaz no tratamento quimioterápico do câncer de mama, ovário e pulmão. Fukumasu et al. (2008) apontam que vários estudos experimentais e epidemiológicos corroboraram que o consumo de algumas plantas pode promover ação quimiopreventiva e/ou antineoplásica. Nos países em desenvolvimento, o largo uso de terapias alternativas ou complementares, como a utilização de plantas medicinais, se confere a maior disponibilidade e acesso, enquanto que nos países desenvolvidos o uso está impulsionado pela preocupação com os efeitos colaterais dos fármacos sintetizados (OMS, 2002; SIMÕES et al., 2002; FUKUMASU et al., 2008).

Pasa et al. (2005) expressam que o conhecimento tradicional sobre o uso de espécies vegetais é amplo, sendo em muitos casos, o único recurso acessível de uma população rural de países em desenvolvimento. Para tanto, resgatando-se este conhecimento e as técnicas terapêuticas têm-se uma maneira de deixar registrado uma forma de aprendizado informal que pode colaborar para uma melhor valorização da medicina popular e, por conseguinte, gerar informações acerca da saúde da comunidade local (PILLA et al.,2006), haja vista que, uma vez perdido, o conhecimento oriundo da cultura popular se torna irrecuperável (ALBUQUERQUE \& ANDRADE, 2002).

Nesta perspectiva, o presente estudo objetivou identificar as plantas medicinais cultivadas em quintais pelos moradores da comunidade rural de Aroeiras, Monsenhor Hipólito-PI, com indicação anticâncer, seja no aspecto preventivo ou terapêutico, buscando-se as formas e partes de usos, além de traçar o perfil socioeconômico dos indivíduos envolvidos na pesquisa, para assim registrar e valorizar as informações acerca do tema em foco, contribuindo desta maneira ao enriquecimento da abordagem acadêmica no âmbito etnobotânico das comunidades tradicionais.

\section{Materiais e Método}

\section{1. Área de estudo}

Monsenhor Hipólito-PI, Brasil, compreende uma área territorial de $401,33 \mathrm{~km}^{2}$ onde está inserida uma população de 7391 habitantes, com predominância da população na zona rural (52,8\%) (IBGE, 2010). Compreendendo o Território de Desenvolvimento Vale do Guaribas, o município encontra-se geograficamente localizado às coordenadas de $06^{\circ} 59^{\prime} 47^{\prime \prime}$ de latitude sul e $41^{\circ} 01^{\prime} 47^{\prime \prime}$ de longitude oeste de Greenwich, tendo como áreas limítrofes ao norte o município de Pio IX, ao sul Campo Grande do Piauí, Francisco Santos a oeste e a leste Alagoinhas do Piauí e Campo 
Grande do Piauí, distando cerca de 369 Km da capital Teresina (AGUIAR \& GOMES, 2004).

O município apresenta um misto no seu extrato vegetal, coma presença de floresta caducifólia e/ou floresta sub-caducifólia/cerrado com transições vegetais de caatinga hiperxerófila e/ou cerrado sub-caducifólio/floresta sub-caducifólia. Quanto ao relevo, a região compreende, principalmente, superfícies tabulares reelaboradas (chapadas baixas), relevo plano com partes suavemente onduladas e altitudes variando de 150 a 300 metros; superfícies tabulares cimeiras (chapadas altas), com relevo plano, altitudes de 400 a 500 metros, com grandes mesas recortadas e superfícies onduladas com relevo movimentado, encostas e prolongamentos residuais de chapadas, desníveis e encostas mais acentuadas de vales e elevações como serras, morros e colinas, com altitudes de 150 a 500 metros (JACOMINE et al., 1986).

No que se alude à caracterização climática, a sede do município encontra-se a uma altitude de $257 \mathrm{~m}$ acima do nível do mar, apresentando temperaturas mínimas de $25^{\circ} \mathrm{C}$ e máximas de $38^{\circ} \mathrm{C}$ (AGUIAR\& GOMES, 2004), com ocorrência de um clima tropical semiárido e quente.

Dada a predominância dos munícipes nos ambientes rurais, o cenário foco do estudo foi a comunidade rural de Aroeiras (figura 1), a qual dista em torno de $13 \mathrm{~km}$ da sede municipal, onde conforme os dados do Programa de Saúde da Família do ano de 2014, apresenta 127 domicílios com 735 pessoas aproximadamente. A escolha deste ambiente deu-se principalmente pela ampla distribuição da área e pelo fácil acesso, uma vez que é cortada pelas rodovias PI-229 e pela BR-020, assim como pela forte cultura de cultivo de plantas em quintais pelos moradores locais.

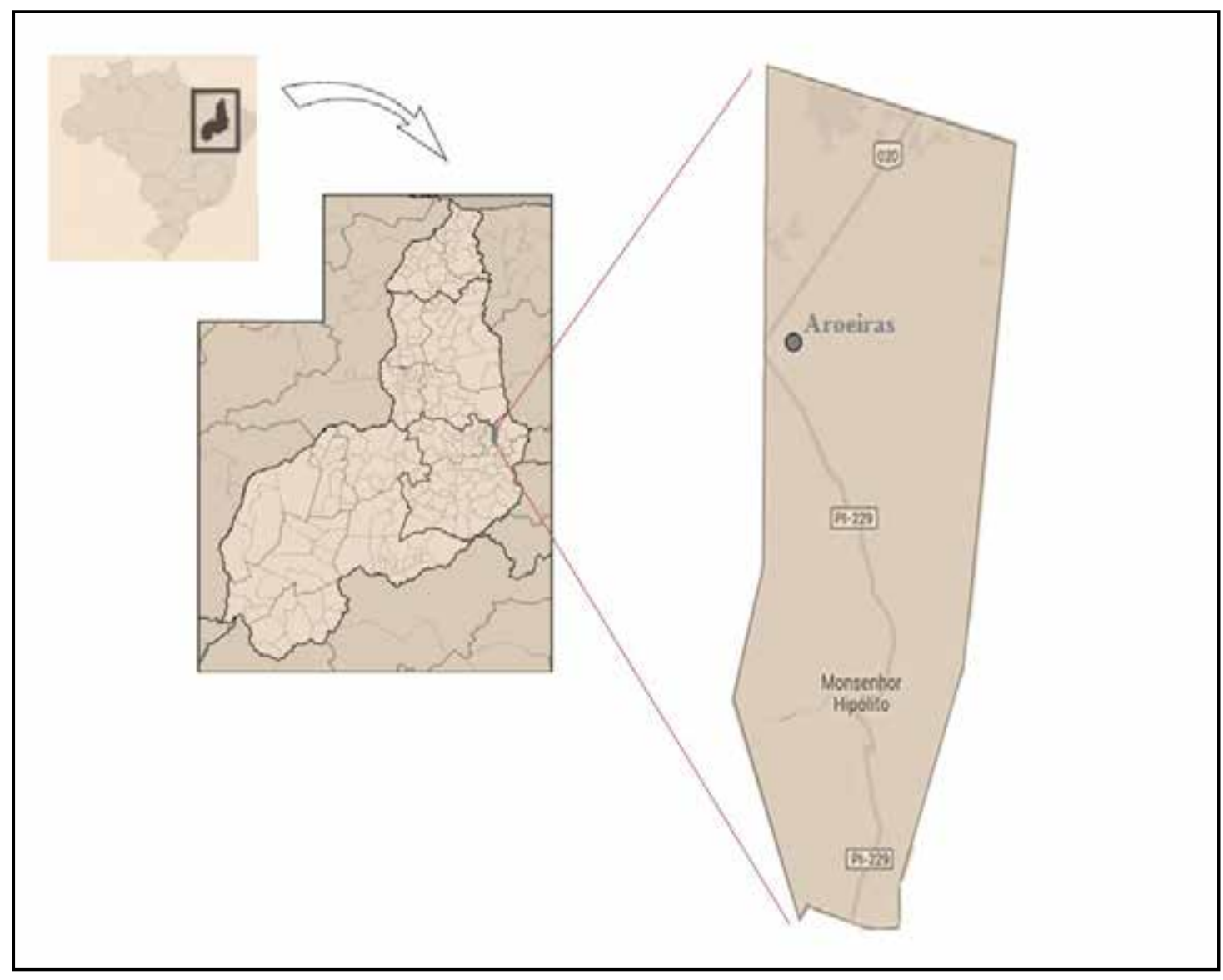

Figura 1- Localização da comunidade rural de Aroeiras, município de Monsenhor Hipólito, Piauí, Brasil Fonte: Autor da pesquisa

Organizada basicamente no grupo familiar, a comunidade se sobressai na estrutura produtiva principalmente através da agricultura baseada na produção sazonal de feijão, milho e mandioca, assim como da colheita e produção do caju, embora a estiagem ao longo dos anos tenha comprometido substancialmente a produção. Combinado a isto, a criação de animais como porcos, galinhas e o gado é também bastante desenvolvida localmente.

\subsection{Coleta e Análise de dados}

Este estudo foi delineado como descritivo, de abordagem quali-quantitativa, cujo trabalho de campo foi realizado no período de fevereiro a maio de 2014, através de visitas em dias irregulares (laborais e em finais de semana) nos 
domicílios familiares da comunidade rural de Aroeiras, mediante entrevistas semi-estruturadas utilizando-se como ferramenta formulários contendo questões abertas (open-ended) e fechadas (closed-ended) (ALBUQUERQUE et al., 2010), contemplando aspectos socioeconômicos bem como informações etnobotânicas sobre as plantas cultivadas nos respectivos quintais, onde apenas um indivíduo por domicílio/quintal foi tomado no estudo.

A seleção dos envolvidos na pesquisa, que foram homens e mulheres acima de 18 anos de idade detentores de conhecimentos acerca do uso e cultivo de plantas, foi feita através da amostragem não-probabilística "bola de neve" (snowball) (BAILEY, 1994), em que um informante indica uma ou mais pessoas que ele acredita ter experiência no assunto abordado.

Para os que cultivavam alguma planta com indicação ao câncer, fez-se uso da técnica da turnê-guiada, no qual o informante foi convidado a fazer uma caminhada pelo quintal durante a entrevista, fornecendo as informações específicas acerca da(s) citada(s)planta(s) (ALBUQUERQUE \& LUCENA, 2004).

Ressalte-se que no processo de coleta de dados foram levadas em consideração as exigências contidas na Resolução 466/12 do Conselho Nacional de Saúde, que regulamenta a pesquisa com seres humanos (BRASIL, 2012). Para isso, os participantes assinaram o Termo de Consentimento Livre e Esclarecido (TCLE) assegurando a estes o anonimato, a privacidade bem como o direito de desistir em qualquer etapa da pesquisa, pesquisa esta que faz parte do projeto analisado e avaliado pelo Comitê de Ética em Pesquisa da Universidade Federal do Piauí (UFPI), sob parecer de apreciação de número 27210114.5.0000.5214.

Junto ao trabalho de levantamento de informações, coletou-se amostras férteis das plantas citadas pelos entrevistados, sempre realizando registro fotográfico das mesmas. As informações pertinentes dos vegetais coletados foram registradas em diário de campo. As etnoespécies coletadas foram herborizados segundo as técnicas propostas por Mori et al. (1989). O material botânico processado e identificado foi incorporado ao acervo do futuro Herbário de Picos assim como duplicatas foram enviadas para o acervo do Herbário Graziela Barroso (TEPB).

Através da base de dados do sítio do Missouri Botanical Garden (www.tropicos.org) e de acordo com o site do The Internacional Plant Names Index - IPNI (www.ipni.org), foram feitas as correções dos nomes dos táxons, assim como, foram adquiridas as abreviaturas dos nomes dos autores. O sistema taxonômico adotado foi o APG IV (THE ANGIOSPERM PHYLOGENY GROUP, 2016).

Quanto à sistematização dos dados obtidos, estes foram organizados em planilhas eletrônicas do Microsoft Excel 2010, para que assim pudesse-se proceder à análise quantitativa das informações. Aos dados foi efetuada a estatística descritiva através de frequências absolutas e percentuais.

\section{Resultados e Discussão}

Foram pesquisados 71 quintais rurais da referida comunidade, consistindo em uma amostra de 71 entrevistados, onde destes $12,68 \%$ eram do sexo masculino e $87,32 \%$ do feminino, compreendida assim a amostra em sua maior parte por mulheres, como observado em vários outros estudos de levantamentos etnobotânicos de plantas medicinais (PINTO et al., 2006; CALÁBRIA et al., 2008; NASCIMENTO \& GONÇALVES,2013; SILVA et al., 2014).

A maioria (23,94\%) situa-se na faixa etária entre 61 e 70 anos, de modo que 73,24\% apresentam idade igual ou superior a 41 anos. Consoante expõe Vendruscolo e Mentz (2006) a maior parte dos entrevistados encontrase no intervalo etário de 40 a 70 anos de idade. Calábria et al. (2008) relacionam um maior saber concernente às plantas medicinais aos indivíduos de maior idade.

Mais de 70\% (71,83\%) afirmaram ser casados, sendo a agricultura a principal atividade de $95,77 \%$ dos entrevistados. No que tange à escolaridade, 45,07\% apresentam apenas ensino fundamental incompleto, seguidos maiormente de 32,39\% de analfabetos. Em uma pesquisa acerca de plantas venenosas no município de Francisco Santos-PI (SILVA et al., 2014), limite de Monsenhor Hipólito-PI também predominou o ensino fundamental incompleto entre os partícipes. Caetano et al. (2014) em seu trabalho acerca das plantas medicinais utilizadas no município de Largato-SE, com ênfase em pacientes oncológicos, também constataram que a maioria dos entrevistados possuíam nível fundamental incompleto, explicando a situação pela ocupação: lavradores, pelo fato da atividade exigir dedicação física durante o dia, impossibilitando o concílio entre estudo e trabalho.

A maioria das residências que compuseram o universo amostral $(39,44 \%)$ eram constituídas por quatro residentes, predominando a renda familiar mensal de um salário-mínimo (39,44\%). Ademais, 78,87\% dos indivíduos indicaram morar na comunidade a um período acima de dez anos, dos quais 18,31\% estão na região desde que nasceram.

Dos 71 indivíduos tomados neste estudo, a maior parte afirmou não cultivar nenhum vegetal que tenha indicação à prevenção ou tratamento do câncer e, apenas, 38,02\% reportaram ter pelo menos um vegetal cultivado no quintal para tais finalidades, conforme evidenciado na figura 2. 


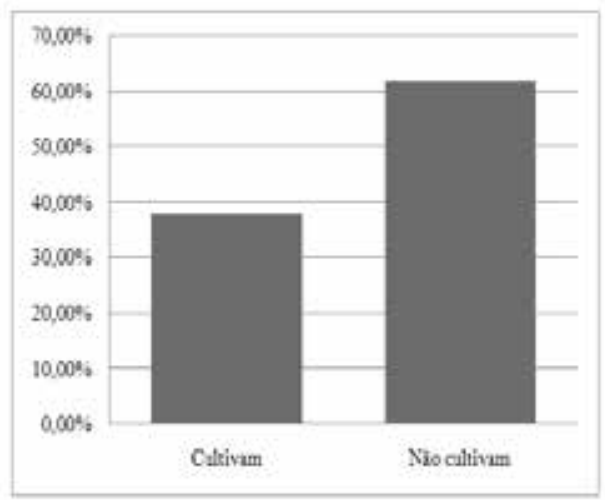

Figura 2. Porcentagem dos entrevistados que afirmaram cultivar ou não alguma planta medicinal anticâncer na comunidade rural de Aroeiras, Monsenhor Hipólito-PI.

Em estudos etnobotânicos realizados por Souza e Felfili (2005); Vendruscolo e Mentz (2006); Pinto et al. (2006); Oliveira et al. (2010); Silva et al. (2012); Santos et al. (2012); Freitas et al. (2012); Aguiar e Barros (2012), Battisti et al. (2013); Alves e Povh (2013); Messias et al. (2015); Araújo e Lemos (2015); Costa e Marinho (2016); Santos et al. (2016) e Souza et al. (2016) figuraram plantas medicinais as quais foram indicadas ao câncer.

Nos quintais pesquisados foram levantadas seis espécies indicadas à prevenção ou tratamento oncológico, as partes utilizadas, bem como a forma de preparo (Tab. 1). Cada espécie pertenceu a uma família botânica diferente.

Através de uma análise de trabalhos etnobotânicos publicados entre a década de 80 até 2008, Melo et al. (2011) apresentam plantas medicinais que foram referidas como anticâncer, em que 84 espécies são citadas como sendo usadas ao tratamento e prevenção do câncer, seja de um modo geral ou contra alguma espécie de tumor específico.

Morinda citrifolia L. e Aloe vera (L.) Burm. f. foram as duas espécies mais representativas no levantamento, ocorrendo, respectivamente, em 55,55\% e 37,03\% das residências que cultivam alguma destas plantas. Capsicum frutescens L. e Chenopodium ambrosioides L. foram indicadas apenas em uma residência (3,70\%).

Oliveira et al. (2014) realizando um estudo sobre o uso de plantas medicinais com a terapêutica anticâncer por pacientes atendidos pela Unidade Oncológica de Anápolis, levantaram 14 espécies que acreditavam poder curar o câncer ou contribuir para tal, onde, semelhantemente, as que mais sobressaíram foram o noni (M. citrifolia), babosa (A. vera) e a graviola (A. muricata). Noni e babosa também figuram no trabalho de Caetano et al. (2015) sobre plantas medicinais utilizadas pela população do município de Lagarto- SE, cujo o noni foi citado como antitumoral, enquanto que a babosa para manejo de reações adversas do câncer.

Solomon (2003) expõe que o suco de noni é bastante difundido na cultura popular como tratamento complementar a uma diversidade de condições médicas, entre elas o câncer. Dixon et al. (1999) e Earle (2001) explica que na farmacopeia tradicional, o fruto do noni é utilizado para prevenir e curar várias doenças, sendo usado principalmente para estimular o sistema imune e assim, para lutar contra infecções bacterianas, virais, parasitárias e fúngicas, do mesmo modo que é também indicado para evitar a formação e proliferação de tumores malignos.

Por outro lado, embora alguns estudos na literatura comprovem a atividade antitumoral de produtos naturais oriundos da M. citrifolia (noni) (BROWN, 2012), ainda não foram encontrados estudos que correlacionassem a eficácia do uso dos chás dessa espécie em pacientes oncológicos, bem como, sua segurança (CAETANO et al., 2015).

No que tange a A. vera, pesquisas realizadas por Lima et al. (2011), Oliveira e Menini Neto (2012), Ferrão et al. (2014) e Costa e Marinho (2016) também trazem a indicação desta espécie ao câncer. Freitas et al. (2014) afirmam que estudos experimentais relatam a atividade antineoplásica da $A$. vera frente a diversas linhagens de câncer, cujo supõe-se que a aloína, aloe-emodina e a acemanana sejam parcialmente responsáveis por essa atividade. Zillmer (2010) também destaca que os estudos já realizados evidenciam o potencial benéfico do uso da Aloe no tratamento do câncer, além dos efeitos já conhecidos, como a ação cicatrizante, amplamente difundida pela medicina popular.

A terceira espécie mais apontada, A. muricata, é também indicada ao câncer em Silva et al. (2012), Santos et al. (2012) e em Alves e Povh (2013). Oberlies et al. (1997) descobriram em seus estudos que a graviola, a partir de extrato das folhas apresenta atividade eficiente no controle de células cancerígenas. Faria (2006) evidenciou que a atividade antitumoral do extrato aquoso das folhas desta planta, quando associado com a doxorrubicina, diminuiu expressivamente o número de tumores em Drosophila melanogaster. Silva e Nepomuceno (2011) afirmam também que outros estudos corroboram a eficiência do extrato das folhas de graviola no auxílio ao tratamento do câncer, todavia, ainda não existem estudos sobre a eficácia do extrato da polpa, com efeito anticarcinogênico.

No que concerne a E. tirucalli, tal espécie foi encontrada em três residências dentre as que apresentavam algum vegetal com indicação ao câncer $(11,11 \%)$, onde, curiosamente, todos os entrevistados foram enfáticos quanto à toxi- 
Tabela 1. Espécies cultivadas em quintais pelos moradores da comunidade rural de Aroeiras, município de Monsenhor Hipólito-PI com indicações à prevenção ou terapêutica do câncer. Convenções: H=Hábito: arv=árvore, her=herbáceo, $\mathrm{arb}=$ arbusto, sub=subarbusto; $\mathrm{Fr} \%=$ frequência percentual da espécie em relação às residências que cultivavam tais plantas.

\begin{tabular}{|c|c|c|c|c|c|c|}
\hline Espécie & $\begin{array}{l}\text { Nome } \\
\text { vulgar }\end{array}$ & Família & $\begin{array}{c}\text { Parte } \\
\text { utilizada }\end{array}$ & $\begin{array}{c}\text { Modos de } \\
\text { preparo e uso }\end{array}$ & $\mathbf{H}$ & Fr\% \\
\hline $\begin{array}{l}\text { Chenopodium } \\
\text { ambrosioides L. }\end{array}$ & $\begin{array}{l}\text { Mastruz, } \\
\text { mentruz }\end{array}$ & Amaranthaceae & folha & $\begin{array}{c}\text { Processar no } \\
\text { liquidificador } \\
\text { ao leite; infusão. }\end{array}$ & her & $3,70 \%$ \\
\hline $\begin{array}{c}\text { Annona } \\
\text { muricata L. }\end{array}$ & Graviola & Annonaceae & $\begin{array}{l}\text { folha, fruto, } \\
\text { sementes }\end{array}$ & $\begin{array}{c}\text { Infusão das folhas ou } \\
\text { sementes; } \\
\text { suco do fruto; } \\
\text { fruta in natura. }\end{array}$ & $\operatorname{arv}$ & $14,81 \%$ \\
\hline $\begin{array}{l}\text { Euphorbia } \\
\text { tirucalli L. }\end{array}$ & $\begin{array}{l}\text { Cachorro- } \\
\text { pelado, } \\
\text { avelós }\end{array}$ & Euphorbiaceae & látex & $\begin{array}{c}\text { Gotas do látex } \\
\text { dissolvidas } \\
\text { em água (2 ou } 3) .\end{array}$ & arb & $11,11 \%$ \\
\hline $\begin{array}{l}\text { Morinda } \\
\text { citrifolia L. }\end{array}$ & Noni & Rubiaceae & fruto & $\begin{array}{c}\text { Suco do fruto associado } \\
\text { a mel, vinho, suco de } \\
\text { uva; } \\
\text { Colocar o fruto seco } \\
\text { fatiado de molho em } \\
\text { água }\end{array}$ & arb & $55,55 \%$ \\
\hline $\begin{array}{c}\text { Capsicum } \\
\text { frutescens L. }\end{array}$ & $\begin{array}{l}\text { Pimenta- } \\
\text { malagueta }\end{array}$ & Solanaceae & fruto & Associado à comida. & sub & $3,70 \%$ \\
\hline $\begin{array}{l}\text { Aloe vera (L.) } \\
\text { Burm. f. }\end{array}$ & Babosa & Asphodelaceae & $\begin{array}{c}\text { folha } \\
\text { (mucilagem) }\end{array}$ & $\begin{array}{c}\text { Processar no } \\
\text { liquidificador } \\
\text { a mucilagem com mel; } \\
\text { pílulas feitas com a } \\
\text { mucilagem com polvilho } \\
\text { de mandioca }\end{array}$ & her & $37,03 \%$ \\
\hline
\end{tabular}

cidade da planta, indicando o uso de poucas gotas do látex (no máximo três) em um litro d'água para o tratamento do câncer, haja vista poder corroer o estômago e/ou intestino, ou até mesmo levar a óbito em grandes quantidades. Cenário semelhante foi mostrado por Oliveira et al. (2010) em um levantamento de plantas medicinais em comunidades rurais do município de Oeiras-PI e por Vieira (2008) no Quilombo dos Macacos em São Miguel do Tapuio, em que o látex da planta foi citado como tóxico caso seja empregado em quantidades maiores que duas gotas.

Baloch e Baloch (2010) realçam que na medicina popular, o látex extraído de qualquer parte das plantas do gênero Euphorbia é considerado tóxico e que muitos estudos feitos com a E. tirucalli mostraram que esta espécie apresenta várias substâncias tóxicas, bem como enzimas proteolíticas. Entretanto, malgrado o risco toxicológico tão tratado no meio científico, o uso do látex desta espécie como tratamento complementar de neoplasias é bem notório em todas as regiões brasileiras, sobretudo no Nordeste, possivelmente por ser esta a região onde este vegetal se propaga com mais facilidade em decorrência das condições climáticas a ele favoráveis (VARRICCHIO et al., 2008a.).

Além disto, o látex deste vegetal também é popularmente conhecido por possuir propriedade antiescorpiônica e ofídica, purgativo, (quando ingerido em alta concentração), antirreumático, antiasmático, antiespasmódico, antibiótico, antibacteriano, antivirótico, expectorante, fungicida, antissifílico, além de resolutivo na terapêutica de carcinomas e epiteliomas benignos (caso ingerido diluído), bem como cauterizante de verrugas (quando utilizados externamente) (FONSECA, 2010). Conforme Varricchio et al. (2008b), a ingestão de altas doses do látex diluído em 
água pode acarretar vasoconstrição, hipóxia tecidual e aumentar a coagulação sanguínea em decorrência da ativação na produção de plaquetas, levando assim ao surgimento de trombos, e a longo prazo levar a danos hepáticos.

Chenopodium ambrosioides, citado em apenas uma residência, também teve indicação ao combate do câncer em uma comunidade rural do município de Caicó-RN, numa pesquisa acerca do uso e diversidade de plantas medicinais da Caatinga (ROQUE et al., 2010). Santos et al. (2012) inventariando as plantas utilizadas como medicinais na comunidade Castanho de Baixo, município de Queimadas na Paraíba também descreveram a indicação desta planta ao câncer. Em estudos in vitro foi descrito que a utilização de extrato de plantas inteiras de C. ambrosioides tem capacidade de matar células de câncer hepático humano e com o óleo essencial, intensa atividade antitumoral em distintas células cancerosas (ITF, 2008).

No que alude à pimenta-malagueta (C. frutescens), ocorrida também em apenas uma residência (3,70\%), o uso desta na forma de molho associado à comida foi relatado como eficiente à prevenção do câncer, divergindo das demais espécies citadas, as quais foram indicadas ao combate e/ou tratamento desta enfermidade. Santos et al. (2008) reporta que as pimentas picantes são utilizadas pela indústria farmacêutica e também pela indústria de cosméticos, trazendo benefícios para a saúde por sua atividade antioxidante e anticancerígena. Alguns cientistas evidenciam que o consumo regular de pimentas pertencentes ao gênero Capsicum provou ter utilidade em várias condições alteradas, como no diabetes, artrites e câncer (AHUJA, 2006).

Observações populares concernentes ao uso e a eficácia de plantas medicinais contribuem de forma positiva para a divulgação das virtudes terapêuticas dos vegetais prescritos com frequência pelos efeitos medicinais que produzem, apesar de não terem, muitas vezes, suas propriedades farmacológicas conhecidas (MACIEL et al., 2002), logo, é evidente a necessidade de estudos que busquem demonstrar a aplicação segura das plantas usadas na medicina tradicional (SOUZA et al., 2016).

Diante do conhecimento tradicional acumulado ao longo das gerações, surge uma necessidade de estudar a utilização das plantas pelos grupos humanos das mais diversas culturas, a fim de obter conhecimentos que possam ser aplicados na busca de substâncias biologicamente ativas para uso na produção de medicamentos (COUTINHO et al., 2002). Todavia, de acordo com Agra et al. (2008) observa-se que os saberes acerca das práticas curativas tradicionais, principalmente do uso plantas silvestres, está desaparecendo em decorrência sobretudo da rápida modernização e da mudança de estilos para os mais contemporâneos, necessitando assim serem resgatados e documentados, visto que, caso tais informações se percam, não será possível a recuperação destas.

Mesmo assim, saliente-se que apesar das benesses que as plantas medicinais podem proporcionar, Oliveira et al. (2014) chamam a atenção que a utilização de vegetais em pacientes com doenças crônicas podem acarretar o agravamento do quadro clínico, posto as possíveis reações colaterais e interações medicamentosas, como por exemplo em pacientes oncológicos. O uso deles simultâneo ao tratamento oncológico convencional pode trazer sérios problemas, uma vez que os medicamentos antineoplásicos em sua maior parte apresentam baixo índice terapêutico e podem interagir com as plantas medicinais, agravando os efeitos adversos.

Junior et al. (2005) afirmam que, aqui no Brasil, o consumo de plantas medicinais tem como característica o uso empírico baseado no senso comum com poucas comprovações científicas adequadas. Além do que, outros fatores como: intoxicação, reações alérgicas, ineficácia no tratamento, podem ser pautados ao uso impróprio dessas plantas, além de erros na identificação das espécies consumidas ou à maneira de como são cultivadas, colhidas, armazenadas, conservadas ou preparadas, resultando em um uso irracional de vegetais ditos medicinais.

As fontes de princípios ativos mais importantes do globo residem na diversidade de plantas com propriedades medicinais, por isso, levantamentos etnofarmacológicos são relevantes ao conhecimento e estudo de espécies com finalidades medicinais (MING, 1996). Houghton et al. (2007) informam que nas últimas décadas, uma expressiva parte das pesquisas para descoberta de novos fármacos está voltada à busca de agentes potenciais para prevenção ou tratamento do câncer e, acerca disto, Lima et al. (2006) acrescentam que os compostos naturais têm sido uma importante fonte destes agentes, visto que muitos vegetais apresentam propriedades anticâncer admiráveis.

\section{Considerações Finais}

Deste modo, percebe-se a importância de estudos etnobotânicos frente aos levantamentos de espécies e, sobretudo, acerca das medicinais, uma vez que possibilita o resgate e a documentação do conhecimento tradicional, saber este que pode fazer suscitar novas pesquisas e avanços ao meio científico, subsidiando à produção de novos fármacos para a terapêutica de inúmeras doenças, dentre elas, o câncer.

Neste ínterim, percebe-se que a conservação do conhecimento tradicional tem valor inestimável para as comunidades locais, uma vez que estes saberes que foram reunidos e repassados ao longo das gerações proporcionam benefícios para estas, mesmo considerando os efeitos colaterais que podem ocasionar, como no caso do uso à revelia das plantas medicinais. 
Voltando-se à comunidade rural de Aroeiras, mesmo diante da modernidade, ainda persiste a cultura do cultivo de vegetais com finalidades terapêuticas nos quintais, relevante forma de manejo da terra, funcionando positivamente tanto no aspecto sociocultural quanto no sustentável e, com base nas plantas cultivadas anticâncer, esclarecimentos sobre o perigo em utilizar ou conciliar tratamento convencional com o medicinal, ou mesmo o uso indiscriminado de tais vegetais se torna imprescindível, para que assim resultados contraproducentes sejam evitados.

\section{Agradecimentos}

Aos moradores da comunidade rural de Aroeiras pela gentileza em contribuir com este trabalho, fornecendo informações imprescindíveis ao desenvolver deste.

\section{Referências}

Aguiar LCGG, Barros RFM. Plantas medicinais cultivadas em quintais de comunidades rurais no domínio do cerrado piauiense (Município de Demerval Lobão, Piauí, Brasil). Revista Brasileira de Plantas Medicinais. 2012; 14(3): 419-434.

Aguiar RB, Gomes JRC. (Org.). Projeto cadastro de fontes de abastecimento por água subterrânea, estado do Piauí: diagnóstico do município de Monsenhor Hipólito. Fortaleza: CPRM - Serviço Geológico do Brasil, 2004.

Ahuja KD. Effects of chilli consumption on postprandial glucose, insulin, and energy metabolism. Journal of Clinical Nutrition. 2006; 84:63-69.

Albuquerque UP, Andrade LHC. Conhecimento botânico tradicional e conservação em uma área de caatinga no estado de Pernambuco, Nordeste do Brasil. Acta Botanica Brasilica. 2002; 16(3):273-285.

Albuquerque UP, Lucena RFP. Métodos e técnicas para a coleta de dados. In: Albuquerque UP, Lucena RFP (editors). Métodos e técnicas na pesquisa etnobotânica. Recife: NUPEEA, 2004. p. 37-62.

Albuquerque UP, Paiva RF, Alencar NL. Métodos e técnicas para coleta de dados etnobiológicos. In: Albuquerque UP, Lucena RFP, Cunha LVFC. (eds.). Métodos e técnicas na pesquisa etnobiológica e etnoecológica. Recife: NUPPEA, 2010. p.39-64.

Alves GSP, Povh JA. Estudo etnobotânico de plantas medicinais na comunidade de Santa Rita, Ituiutaba - MG. Biotemas. 2013; 26(3):231-242.

Amorozo MCM. Uso e diversidade de plantas medicinais em Santo Antônio do Leverger, MT, Brasil. Acta Botanica Brasilica. 2002; 16(2):189-203.

APG IV (Angiosperm Phylogeny Group). An update of the Angiosperm Phylogeny Group classification for the orders and families of flowering plants: APG IV. Botanical Journal of the Linnean Society. 2016; 181(1):1-20.

Araújo EC, Oliveira RAG, Coriolano AT, Araújo EC. Uso de plantas medicinais pelos pacientes com câncer de hospitais da rede pública de saúde em João Pessoa (PB). Revista Espaço para a Saúde. 2007; 8(2):44-52.

Araújo JL, Lemos JR. Estudo etnobotânico sobre plantas medicinais na comunidade de Curral Velho, Luís Correia, Piauí, Brasil. Biotemas. 2015; 28(2):125-136.

Bailey K. Methods of social research. $4^{\mathrm{a}}$ ed. New York: The Free Press, 1994. 588 p.

Baloch IB, Baloch MK. Irritant and co-carcinogenic diterpene esters from the latex of Euphorbia cauducifolia L. Journal of Asian Natural Products Research. 2010; 12(7): 600-613.

Battisti C, Garlet TMB, Essi L, Horbach RK, Aandrade A, Badke MR. Plantas medicinais utilizadas no município de Palmeira das Missões, RS, Brasil. Revista Brasileira de Biociências,2013; 11(3):338-348. 
BRASIL. Ministério da Saúde. Conselho Nacional de Saúde. Comissão Nacional de Ética em Pesquisa. Normas para pesquisa envolvendo seres humanos: (Res. CNS 466/12 e outros) Brasília, DF, 2012.Acesso em: 19/09/2014.

BRASIL. Ministério da Saúde. Instituto Nacional de Câncer. Estimativa/2010: incidência de câncer no Brasil. Rio de Janeiro: INCA, 2009.

Brown AC. Anticancer activity of Morinda citrifolia (Noni) fruit: a review. Phytotherapy Research 2012;26(10):1427-1440.

Caetano NLB, Ferreira TF, Reis MRO, Neo GGA, Carvalho AA. Plantas medicinais utilizadas pela população do município de Lagarto- SE, Brasil - ênfase em pacientes oncológicos. Revista Brasileira de Plantas Medicinais. 2015; 17(4):748-756.

Caetano RS, Souza ACR, Feitoza LF. O uso de plantas medicinais utilizadas por frequentadores dos ambulatórios Santa Marcelina, Porto Velho - RO. Revista Saúde e Pesquisa. 2014; 7(1): 55-63.

Costa JC, Marinho MGV. Etnobotânica de plantas medicinais em duas comunidades do município de Picuí, Paraíba, Brasil. Revista Brasileira de Plantas Medicinais. 2016; 18(1):125-134.

Coutinho DF, Travassos LMA, Amaral FMM. Estudo etnobotânico de plantas medicinais utilizadas em comunidades indígenas no estado do Maranhão - Brasil. Visão Acadêmica. 2002; 3(1): 7-12.

Dixon AR, Mcmillen H, Etkin NL. Ferment this: the transformation of Noni, a traditional polynesian medicine (Morinda citrifolia, Rubiaceae). Economic Botany. 1999; (53):51-68.

Earle JE. Plantas medicinales en el tropico humedo. Editorial Guayacán, San José. 2001.

Faria MI. Efeito Anticarcinogênico da folha da Graviola (Annona muricata) por meio do teste para detecção de clones de tumor (Warts) em (Drosophila melanogaster), in: Anais do 1ํㅗㄹ Seminário de Iniciação Científica do Programa Institucional de Bolsas de Iniciação Científica do Conselho Nacional de Desenvolvimento Científico e Tecnológico PIBIC/CNPq/UEMG. 2006.

Ferrão BH, Oliveira HB, Molinari RF, Teixeira MB, Fontes GG, Amaro MOF et al. Importância do conhecimento tradicional no uso de plantas medicinais em Buritis, MG, Brasil. Ciência e Natura, 2014;(36):321-334.

Fonseca D, Fioravanti C. As plantas milagrosas. Globo Ciência. 1995.

Fonseca ZA - 2010 - Gêneros vegetais que possuem espécies medicinais. Principais espécies vegetais de cada gênero, 2010.

Freitas AVL, Coelho MFB, Maia SSS, Azevedo RAB. Plantas medicinais: um estudo etnobotânico nos quintais do Sítio Cruz, São Miguel, Rio Grande do Norte, Brasil. Revista Brasileira de Biociências. 2012; 10(1):48-59.

Freitas VS, Rodrigues RAF, Gaspi FOG. Propriedades farmacológicas da Aloe vera (L.) Burm. f.. Revista Brasileira de Plantas Medicinais. 2014; 16(2): 299-307.

Fukumasu H, Latorre AO, Bracci N, Gorniak SL, Dalgi MLZ. Fitoterápicos e potenciais interações medicamentosas na terapia do câncer. Revista Brasileira de Toxicologia. 2008; 21(2):49-59.

Houghton P, Fang R, Techatanawat I, Steventon G, Hylands PJ, Lee CC. The sulphorhodamine (SRB) assay and other approaches to testing plant extracts and derived compounds for activities related to reputed anticancer activity. Methods. 2007; 42(4):377-387.

IBGE - 2010 -Perfil das cidades piauienses. Instituto Brasileiro de Geografia e Estatística. Disponível em: $<$ http://www. ibge.gov.br/cidadesat/xtras/perfil.php?codmun=220650\&search=piauil monsenhor-hipolito $>$. Acesso em 10 Jun. 2014

ITF. Índice Terapêutico Fitoterápico. Petrópolis: Editora de Publicações Biomédicas LTDA, 2008. 328p.

Jacomine PKT, Cavalcanti AC, Pessoa SCP, Burgos N, Melo Filho HFR, Lopes OF et al. Levantamento exploratório reconhecimento de solos do Estado do Piauí. Rio de Janeiro, EMBRAPA-SNLCS/SUDENE-DRN, 1986. 782 p. 
Lima AP, Pereira FC, Vilanova-Costa CAST, Ribeiro ASBB, Silveira-Lacerda EP. Avaliação da atividade antitumoral e citotóxica da planta Siolmatra brasiliensis. Revista Eletrônica de Farmácia. 2006; 3(2): 10-12.

Lima RA, Magalhães SA, Santos MRA. Levantamento etnobotânico de plantas medicinais utilizadas na cidade de Vilhena, Rondônia. Revista Pesquisa \& Criação. 2011; 10(2):165-179.

Lorenzi H, Matos FJA. Plantas medicinais no Brasil: nativas e exóticas. Nova Odessa: Plantarum, 2002. 572p.

Maciel MAM, Pinto AC, Veiga Junior VF, Grynberg NF, Echevarria A. Plantas medicinais: a necessidade de estudos multidisciplinares. Química Nova. 2002; 25(3):429-438.

Melo JG, Santos AG, Amorim ELC, Nascimento SCD, Albuquerque UP. Medicinal plants used as antitumor agents in Brazil: an ethnobotanical approach. Evidence-Based Complementary and Alternative Medicine. 2011; (2011):14.

Messias MCTB, Menegatto MF, Prado ACC, Santos BR, Guimarães MFM. Uso popular de plantas medicinais e perfil socioeconômico dos usuários: um estudo em área urbana em Ouro Preto, MG, Brasil. Revista Brasileira de Plantas Medicinais. 2015; 17(1):76-104.

Ming LC. Coleta de plantas medicinais. In: Stasi DIL (Org.). Plantas medicinais: arte e ciências - um guia de estudo interdisciplinar. São Paulo: UNESP, 1996.

Mori AS, Silva LAM, Lisboa G, Coradin L. Manual de manejo do herbário fanerogâmico. Ilhéus, Porto Alegre, Centro de Pesquisas do Cacau - CEPLAC, 1989. 104 p.

Nascimento APLF, Gonçalves K. Uso de plantas cultivadas em quintais urbanos no município de campina do Monte Alegre, SP. In: Anais do11ํㅡㄹ Congresso de Ecologia do Brasil, Setembro, Porto Seguro-BA, 2013.

Oberlies NH, Chang CJ, McLaughlin JL. Structure-activity relationships of diverse Annonaceous acetogenins against multidrug resistant human mammary adenocarcinoma (MCF-7/Adr) cells. Journal of Medicinal Chemistry. 1997;(40):2102-2106.

Oliveira ER, Menini Neto L. Levantamento etnobotânico de plantas medicinais utilizadas pelos moradores do povoado de Manejo, Lima Duarte - MG. Revista Brasileira de Plantas Medicinais. 2012; 14(2):311-320.

Oliveira FCS, Barros RFM, Moita Neto JM. Plantas medicinais utilizadas em comunidades rurais de Oeiras, semiárido piauiense. Revista Brasileira de Plantas Medicinais. 2010; 12(3):282-301.

Oliveira LAR, Machado RD, Rodrigues AJL. Levantamento sobre o uso de plantas medicinais com a terapêutica anticâncer por pacientes da Unidade Oncológica de Anápolis. Revista Brasileira de Plantas Medicinais. 2014; 16(1):32-40.

Oliveira LAR, Machado RD, Rodrigues AJL. Levantamento sobre o uso de plantas medicinais com a terapêutica anticâncer por pacientes da Unidade Oncológica de Anápolis. Revista Brasileira de Plantas Medicinais. 2014; 16(1): 32-40.

Organización Mundial de la Salud (OMS). Estrategia de la OMS sobre medicina tradicional, 2002-2005. Genebra, Switzerland,2002.

Pasa MC, Soares JJ, Guarim Neto, G. Estudo etnobotânico na comunidade de Conceição-Açu (alto da bacia do rio Aricá Açu, MT, Brasil). Acta Botanica Brasilica. 2005; 19(2):195-207.

Pilla MAC, Amorozo MCM, Furlan A. Obtenção e uso das plantas medicinais no distrito de Martim Francisco, município de Mogi-Mirim, SP, Brasil. Acta Botanica Brasilica. 2006; 20(4):789-802.

Pinto EPP, Amorozo MCM, Furlan A. Conhecimento popular sobre plantas medicinais em comunidades rurais de mata atlântica - Itacaré, BA, Brasil. Acta Botânica Brasílica. 2006; 20(4):751-762.

Roque AA, Rocha RM, Loiola MIB. Uso e diversidade de plantas medicinais da Caatinga na comunidade rural de Laginhas, município de Caicó, Rio Grande do Norte (nordeste do Brasil). Revista Brasileira de Plantas Medicinais. 2010; 12(1):31-42. 
Santos ABN, Araújo MP, Sousa RS, Lemos JR. Plantas medicinais conhecidas na zona urbana de Cajueiro da Praia, Piauí, Nordeste do Brasil. Revista Brasileira de Plantas Medicinais. 2016; 18(2):442-450.

Santos JAB, Silva GF, Oliveira LC. Avaliação dos Capsaicinóides em Pimentas Malagueta. Revista Eletrônica da FJAV. 2008;(2): 91-100.

Santos SLDX, Alves RRN, Barbosa JAA, Brasileiro TF. Plantas utilizadas como medicinais por uma comunidade do semiárido da Paraíba, Nordeste do Brasil. Revista Brasileira de Farmácia. 2012; 93(1):68-79.

Silva LM, Nepomuceno JC. Efeito modulador da polpa da graviola (Annona muricata) sobre a carcinogenicidade da mitomicina C, avaliado por meio do teste para detecção de clones de tumor (warts) em Drosophila melanogaster. Revista Perquirere. 2011; 1(8):80-94.

Silva LRR, Abreu MC, Ferreira PMP, Pacheco ACL, Calou IBF, Cerqueira GS. Plantas Tóxicas: conhecimento de populares para prevenção de acidentes. RevInter. 2014; 7(2):17-36.

Silva NCB, Regia ACD, Esquibel MA, Santos JES, Almeida MZ. Uso de plantas medicinais na comunidade quilombola da Barra II - Bahia, Brasil. Boletín Latinoamericano y del Caribe de Plantas Medicinales y Aromáticas. 2012; 11(5):435 - 453.

Simões CMO, Schenkel EP, Gosmann G. Farmacognosia: da planta ao medicamento. 4.ed. Porto Alegre: ed. UFRGS, 2002.

Solomon N. Cancer: How and why tahitian noni juice helps in treatment and prevention and noni: nature's gift to cancer patients. Direct Source Publishing: Lachine, QC, Canada. 2003.

Souza CD, Felfili JM. Uso de plantas medicinais na região de Alto Paraíso de Goiás, GO, Brasil. Acta Botânica Brasílica. 2006; 20(1):135-142.

Souza LF, Dias RF, Guilherme FAG, Coelho CP. Plantas medicinais referenciadas por raizeiros no município de Jataí, estado de Goiás. Revista Brasileira de Plantas Medicinais. 2016; 18(2):451-461.

Souza LF, Dias RF, Guilherme FAG, Coelho, CP. Plantas medicinais referenciadas por raizeiros no município de Jataí, estado de Goiás. Revista Brasileira de Plantas Medicinais. 2016; 18(2):451-461.

Varricchio MCBN, Sales F, Silva S, Kuster RM, Pyrrho AS, Castelo Branco MLT. Efeitos toxicológicos crônicos do látex bruto de E. tirucalli (Aveloz) sobre peso de fígado e baço conforme o uso tradicional: Um estudo preliminar. Biofar. 2008a;2(2): 16-21.

Varricchio MCBN, Silva S, Gomes NBN, Kuster RM, Lage CLS. O uso de Euphorbia tirucalli (Aveloz) em medicina tradicional e as evidencias cientificas. Biofar. 2008b; 3(1):84-92.

Veiga Junior VF, Pinto AC, Maciel MAM. Plantas medicinais: cura segura? Química Nova. 2005; 28(3): 519-528.

Vendruscolo GS, Mentz LA. Levantamento etnobotânico das plantas utilizadas como medicinais por moradores do bairro Ponta Grossa, Porto Alegre, Rio Grande do Sul, Brasil. Iheringia, Série Botânica. 2006; 61(1-2):83-103.

Viegas Junior C, Bolzani VS, Barreiro EJ. Os produtos naturais e a química medicinal moderna. Química Nova. 2006; 29(2):326-337.

Vieira FJ. Uso e diversidade dos recursos vegetais utilizados pela Comunidade Quilombola dos Macacos em São Miguel do Tapuio. 2008.124p. Dissertação (Mestrado em Desenvolvimento e Meio Ambiente) - Universidade Federal do Piauí, Teresina, 2008.

Zilmer JGV, Arrieira IC, Ceolin T, Heck RM, Barbieri RL. Utilização da babosa no cotidiano de usuários portadores de Câncer. Revista Baiana de Saúde Pública. 2010; 34(4):773-782. 